\title{
La construcción de las bases para la elaboración de mapas de riesgo. Una forma de intervención social desde Terapia Ocupacional
}

\section{Rosana Ariño \\ rosana.arino@gmail.com}

Mariana Boffelli

m_boffelli@yahoo.com.ar

Milagros Demiryi

milagros.demiryi@gmail.com

Daniela Chiapessoni

danielachiapessoni@hotmail.com
Docentes investigadoras de la Facultad de Bioquímica y Ciencias Biológicas. Universidad Nacional de Litoral, Argentina.
Integración de la docencia y la extensión /

Intervenciones

RECEPCIÓN: 24/06/16

ACEPTACIÓN FINAL: 10/10/16

\section{Resumen}

El artículo presenta la experiencia desarrollada por estudiantes que cursaron $1^{\circ}$ y $4^{\circ}$ año de la Licenciatura en Terapia Ocupacional de la Universidad Nacional de Litoral (UNL), junto con agentes municipales, vecinos y organizaciones del barrio Villa Oculta de la ciudad de Santa Fe.

En el marco de la Educación Experiencial, esta práctica de extensión incorporada en el currículo posibilitó a los futuros profesionales la oportunidad de involucrarse en la resolución de problemas reales y complejos de la comunidad.

El trabajo consistió en una experiencia piloto para la construcción de un mapa de riesgo, los que han funcionado como instrumentos puente entre el saber popular, la toma de decisiones y el saber académico. Experiencia posible por el interés de la Dirección de Gestión de Riesgo, vecinos, estudiantes y docentes de la UNL.

Palabras-clave

- Terapia Ocupacional

- Desastres

- Mapas de Riesgo

- Prácticas de Extensión de Educación Experiencial

\section{Resumo}

O artigo apresenta a experiência desenvolvida pelos alunos que frequentam primeiro ano e quarto ano do Bacharelado em Terapia Ocupacional - UNL, juntamente com funcionários municipais, vizinhos e organizações do bairro "Villa Oculta" da cidade de Santa Fe.

Como parte da Educação Experimental, a prática de extensão incorporada no currículo propiciou aos futuros profissionais a oportunidade de participar na resolução de problemas reais e complexos da comunidade.

O trabalho se baseou em um projecto-piloto para a construção de um mapa de risco, que funcionou como uma ponte entre os instrumentos de conhecimento popular, a tomada de decisões e conhecimento acadêmico. A experiência foi possível pelo interesse da Diretoria de Gestão de Risco, vizinhos, estudantes e professores da UNL.

Palavras-chave

- Terapia Ocupacional

- Desastres

- Mapeamento de Riscos

- Práticas de Extensão de Educação Experiencial
Para citación de este artículo

Ariño, R.; Bofelli, M.; Demiryi, M. y Chiapessoni, D. (2016). La construcción de las bases para la elaboración de mapas de riesgo. Una forma de intervención social desde Terapia Ocupacional. En Revista +E versión digital, (6), pp. 208-215. Santa Fe, Argentina: Ediciones UNL. 


\section{Introducción}

Nos parece oportuno dar a conocer la disciplina a la que pertenecemos, que es Terapia Ocupacional (TO), la carrera corresponde a la Facultad de Bioquímica y Ciencias Biológicas de la Universidad Nacional del Litoral (FCB-UNL) y articula un conjunto de conocimientos, técnicas, procedimientos que centralizan su atención en la ocupación del hombre y la importancia del significado que éste le atribuye. Tal como lo sostienen Fischer y Pierce (1998):

"las ocupaciones no son actividades aisladas, sino que están conectadas por una red de actividades diarias que ayudan a las personas a cumplir sus necesidades básicas y contribuir a las necesidades de su familia, de sus amigos y de la comunidad. La práctica centrada en la ocupación se interesa por las ocupaciones significativas seleccionadas por las personas y realizadas en sus ambientes típicos". (citado por Blesedell; Scohn; Boyt, 2005:29)

El diseño del plan de estudios, y consecuentemente de las prácticas profesionales contempladas en el él, está basado en los lineamientos de las normas mínimas revisadas para la formación de terapeutas ocupacionales de la Federación Mundial de Terapeutas Ocupacionales (WFOT). Estas normas hacen referencia a la variedad de los escenarios y las poblaciones en las que se realizan las prácticas, a la supervisión, la evaluación y la duración de las mismas, de las cuales esta última contempla más de 1000 horas. Las prácticas profesionales se encuentran presentes en todo el trayecto de la carrera y promueven así una mejor formación para el ejercicio profesional del futuro graduado. Estas prácticas promueven una articulación vertical y horizontal de las asignaturas y son supervisadas por docentes a cargo de las cátedras y tutores institucionales.

A partir de aquí consideramos destacar la relevancia de reconocer como necesidad nodal que el estudiante pueda realizar un recorrido durante el cursado de su carrera en los diferentes escenarios complejos y cambiantes e inherentes a la realidad cotidiana a nivel local, regional, nacional e internacional; ya que desde la creación de la carrera existen experiencias en las que los estudiantes pueden llevar adelante sus prácticas en los ámbitos de salud, educación y promoción comunitaria en nuestro país y en otros países (esto último es posible a través de los programas internacionales de movilidad estudiantil que promociona nuestra Universidad). Después de haber dado a conocer sintéticamente la disciplina de la que partimos y los intereses que persigue, consideramos relevante tomar el aporte de Alicia De Alba (1995) cuando señala el concepto de noción de curriculum como práctica social.

La autora sostiene:

"Por curriculum se entiende a la síntesis de elementos culturales (conocimientos, valores, costumbres, creencias, hábitos) que conforman una propuesta político-educativa pensada e impulsada por diversos grupos y sectores sociales cuyos intereses son diversos y contradictorios, aunque algunos tienden a ser dominantes o hegemónicos, y otros tienden a oponerse y resistirse a tal dominación o hegemonía”. (1995:59)

Teniendo en cuenta lo dicho, creemos que está en congruencia con lo que la UNL a partir de su Plan de Desarrollo Institucional (2010-2019) plantea cuando propone:

"Una Universidad que genere y gestione propuestas académicas dinámicas, flexibles y de calidad destinadas a formar ciudadanos críticos, con sólida formación profesional, aptitud emprendedora, competencias para un desempeño internacional y compromiso social para integrarse a una sociedad democrática; que se proponga ampliar las fronteras del conocimiento en un adecuado equilibrio entre la investigación disciplinar, la interdisciplinaria y la orientada a problemas con sentido ético y al servicio de la sociedad y el país; que extienda sus investigaciones y enseñanzas al entorno social e interactúe con éste para fomentar la interculturalidad y asegurar que sus miembros mantengan arraigo, se interesen por las problemáticas locales y contribuyan a solucionar". (2010:14) 
Donde también se destaca que:

"la extensión es una función esencial de nuestra Universidad que promueve el diálogo permanente con el Estado -en sus diferentes jurisdicciones-, con otras instituciones educativas y científico tecnológicas del sistema provincial y nacional, con las organizaciones de la sociedad civil y de la producción en la búsqueda de soluciones a los problemas del contexto, lo que confiere a estas acciones una alta pertinencia social".

Y se hace alusión a que:

"la integración entre la extensión y la enseñanza nos posiciona desde una perspectiva en la que los saberes culturales y los conocimientos académicos-científicos se ponen en juego con otros saberes y conocimientos que posee la sociedad, lo que posibilita analizar y abordar los problemas a partir de una concepción y comprensión de la complejidad”. (2010:20)

En este sentido, este artículo pretende poner en conocimiento lo que ha posibilitado la práctica de extensión de educación experiencial realizada con una asignatura de $1^{\circ}$ año y otra de $4^{\circ}$ de la carrera, en el marco de las convocatorias de Prácticas de Extensión de Educación Experiencial llevadas a cabo por la Secretaría de Extensión de esta Universidad y en consonancia con las oportunidades de prácticas preprofesionales que también ofrece el plan de estudio de la carrera.

Esta Práctica de Extensión de Educación Experiencial, titulada "Experiencia piloto para la construcción de un mapa de riesgo", orientó a conformar un espacio colaborativo de trabajo en TO.

El diseño de la propuesta inicialmente fue pensado para darle la posibilidad a los estudiantes, de aprender a través de la experiencia, de manera diferente, contenidos disciplinares de la carrera.

En términos de David Kolb (1984), el aprendizaje es el proceso por el cual el conocimiento es creado a través de la transformación de la experiencia. De modo que la experiencia rutinaria es diferente de la acción. La experiencia es aprendizaje cuando el sujeto transforma la situación. Por lo tanto, podríamos decir que la educación experiencial genera cambios. Esta noción se sustenta en las ideas de Dewey, para quien la construcción de conocimiento se da a partir de la experiencia concreta.

Teniendo en cuenta esta definición, planteamos una propuesta con el fin de realizar un aporte a la formación profesional de los estudiantes universitarios, en especial a los que están cursando las asignaturas de la carrera de Licenciatura en TO, a los profesionales y agentes municipales y a la población con quien se trabajó la misma. Este tipo de experiencia creemos que posibilita a los futuros profesionales la oportunidad de involucrarse en la resolución de problemas reales de la comunidad, implica trabajar en escenarios complejos, con problemas que se resisten a ser abordados desde una única perspectiva disciplinar. Es por esto que el trabajo realizado fue en el marco de la educación

experiencial que, en términos de la Prof. Alicia Camilloni (2010), en su conferencia "Las prácticas académicas en espacios socio culturales, productivos y/o comunitarios. Pensando la educación experiencial", significa:

"una estrategia de enseñanza con enfoque holístico, que está destinada a relacionar el aprendizaje académico con la vida real. Con ese fin, propone al alumno realizar actividades en las que, a partir de esa conexión con la práctica, desarrollarán experiencias que resultan de poner a prueba en situación auténtica las habilidades y los conocimientos teóricos que poseen, evaluarán sus consecuencias, enriquecerán esos conocimientos y habilidades e identificarán nuevos problemas". ${ }^{1}$

\section{Antecedentes, actividades y abordajes de la temática desde el TO}

La Práctica de Extensión de Educación Experiencial sobre mapa de riesgo tiene anclaje en el proyecto de investigación "Análisis de mapas de riesgo disponibles en organismos oficiales de la ciudad de Santa Fe y de la percepción del riesgo por parte de los pobladores. Un aporte a la construcción de ciudades resilientes". Desde este proyecto de investigación se desprendió el contacto con los agentes municipales de la Dirección de Riesgo de la Municipalidad de la ciudad de Santa Fe, y a partir de él se logró establecer una línea de trabajo en conjunto sobre mapas de riesgo. Este equipo de docentes investigadores y extensionistas viene trabajando desde el año 2005 en torno a la temática de desastres desde la perspectiva ocupacional, habiendo desarrollado hasta el momento numerosas actividades relacionadas, entre ellas tres proyectos de investigación acreditados por la UNL: Curso de Acción para la Investigación y Desarrollo (en adelante CAI+D) 2005, "La modificación de los hábitos y rutinas diarias de personas afectadas por la catástrofe hídrica"; CAl+D 2009, "Diseño, desarrollo y evaluación de un curso de capacitación virtual sobre intervención en catástrofes" y CAl+D 2011, actualmente en curso, mencionado con anterioridad.

En mayo de 2010 en la ciudad de Santiago de Chile, en el marco del XV Congreso de la Federación Mundial de TO, el equipo fue convocado a tomar parte como coordinador de las mesas de discusión del workshop de la Federación Mundial de Terapeutas Ocupacionales titulado: "Preparación y respuesta ante Desastres y Terapia Ocupacional”, y en el taller para intervención en catástrofe realizado poscongreso exclusivamente para los TO chilenos.

1) Se trata de la transcripción de la Conferencia dictada por Camilloni, A. (2009). "Las prácticas académicas en espacios socio culturales, productivos y/o comunitarios. Pensando la educación experiencial", en el marco del Curso de posgrado "La resolución de problemas abiertos como estrategia de enseñanza en la universidad. Las prácticas académicas en espacios socio culturales, productivos y/o comunitarios". Facultad de Humanidades y Ciencias. UNL. 10 de junio de 2009. 

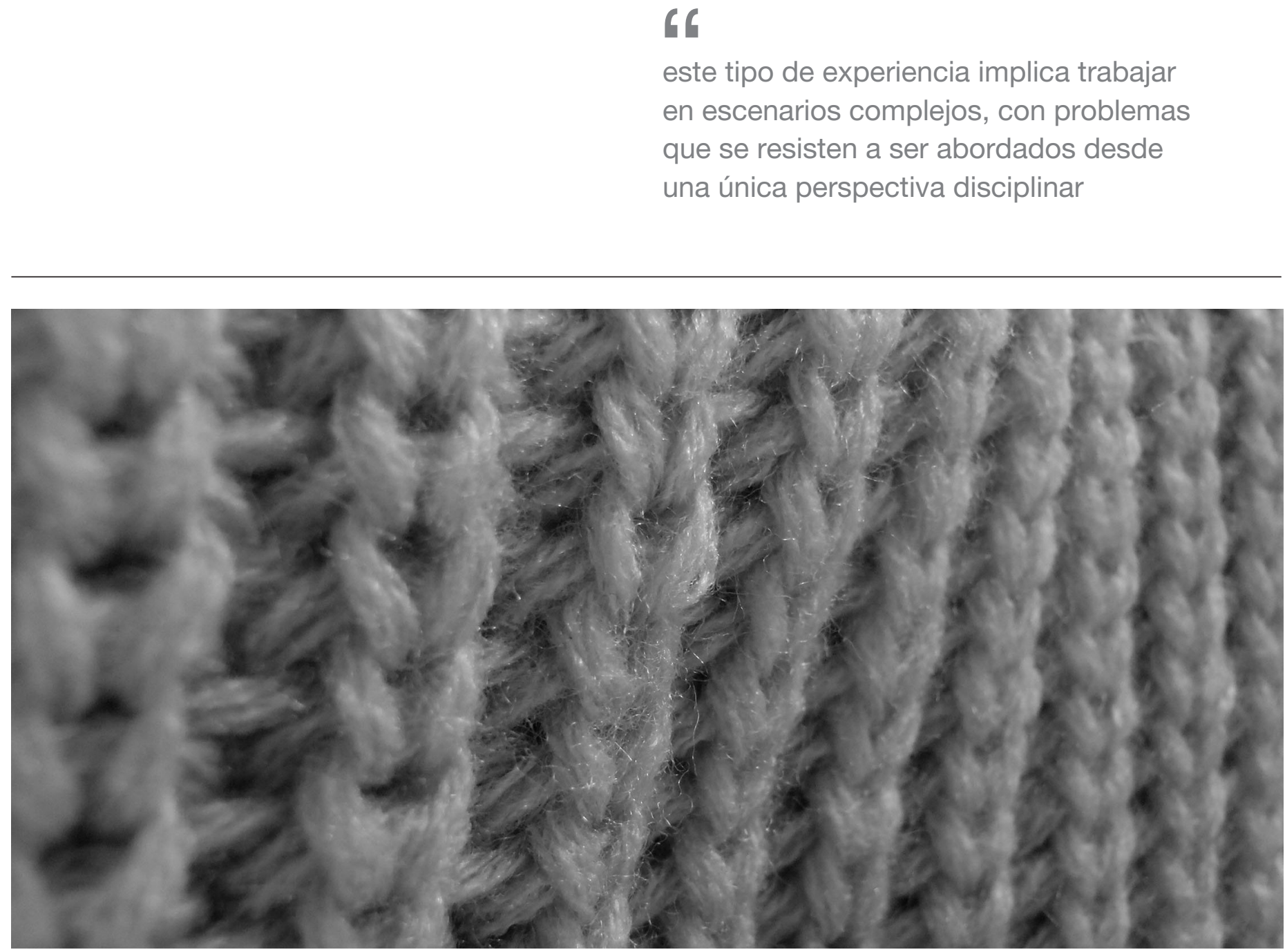

(c) Hugo Pascucci

Asimismo, este equipo ha realizado presentaciones en cursos, jornadas y congresos locales, nacionales e internacionales; publicaciones en revistas nacionales y extranjeras; cursos de capacitación; formación de posgrado; creación de la Red Latinoamericana y del Caribe TO en alerta (en el XII Congreso Brasilero y IX Congreso Latinoamericano de Terapia Ocupacional, octubre de 2011, San Pablo, Brasil); elaboración de una propuesta curricular que incorpora el tema desastres en la formación de grado de las carreras que se dictan en la Escuela Superior de Sanidad; actividades de difusión, asesoramiento, etcétera.

Tal como se puede apreciar en el recuento de las actividades desarrolladas, hay acciones propias de la investigación y la extensión que producen nueva evidencia. Desde los inicios, surge como una premisa básica del equipo de trabajo la necesidad de compartir conocimiento no solo como forma de cumplimentar instancias formales del plan de trabajo, sino, y fundamentalmente, en la convicción de que la universidad debe servir a la comunidad, de la cual forma parte. El conocimiento que se comparte multiplica su valor y permite transformarlo en una instancia de acción donde la experiencia desarrollada pueda plasmarse en acciones concretas del quehacer profesional. Los pasos dados por el equipo confirmaban la necesidad de construir espacios de intercambio y formación accesibles a toda la comunidad profesional y estudiantil interesada en el área. Uno de los objetivos centrales planteados desde la primera línea de investigación se proponía contribuir con la formación de recursos en el área de investigación de Terapia Ocupacional y con la producción de aportes curriculares que cualifiquen la formación de los futuros profesionales del área de salud en general y de Terapia ocupacional, en particular. Se pretendía socializar los resultados de las investigaciones y acciones relacionadas con las situaciones de desastres, entendiendo que la tarea de investigar trasciende la meta de construir conocimiento. Esta necesidad se presentó como evidente al equipo al detectar ausencia de la temática en los programas académicos de la profesión y como demanda concreta de profesionales y alumnos en congresos de TO. 
Entre las primeras ofertas de capacitación se cuentan dos cursos dictados en el ámbito de la UNL, uno para graduados y otro para alumnos de la Licenciatura en TO sobre desastres, vulnerabilidad e intervenciones de terapistas ocupacionales en situación de emergencia. $^{2}$

En el segundo trabajo de investigación el equipo encaró su más ambicioso proyecto de formación: desarrollar e implementar un curso de modalidad virtual de formación de terapistas ocupacionales en intervención en emergencias y desastres. El mismo se ofreció a profesionales de Latinoamérica y el Caribe y se dictó en el campus virtual de la UNL. En la preparación del curso se trabajó conformando un equipo interdisciplinario con integrantes del Centro Multimedial de Educación a Distancia y se definieron temas relevantes. Se le dio por nombre "Intervención de TO en emergencias y desastres" (2013) y contó con participantes de diversos países de la región y docentes invitados. Se encontró en este proyecto el punto de partida para desarrollar y validar herramientas de formación de recursos humanos para la intervención ocupacional en situaciones de desastre.

En esta línea de formación y capacitación, otros cursos y seminarios permitieron al equipo acercarse a diferentes comunidades académicas y profesionales. ${ }^{3}$

Como profesionales sociosanitarios consideramos que no podemos eludir la responsabilidad social de intervenir desde nuestro campo disciplinar. Como docentes, investigadores y extensionistas nos proponemos apostar al desarrollo del conocimiento científico en relación con los objetos de estudio de TO para abrir camino en el desarrollo de teoría y práctica en un área emergente de la disciplina, en la que gran parte está por hacerse.

\section{El riesgo: las inundaciones en la ciudad de Santa $\mathrm{Fe}$}

En la última década, el incremento, la frecuencia y la intensidad de desastres ocurridos en distintas partes del mundo, han afectado el bienestar, la salud y la seguridad de personas y comunidades. Pérdidas y daños constituyen evidencias empíricas de los desastres que indefectiblemente están asociados al riesgo como condición pre-existente.

La ciudad de Santa Fe abarca $268 \mathrm{~km}^{2}$, de los cuales el $70 \%$ está ocupado por ríos, lagunas y bañados, totalizando un área urbana de 187,6 km². Limitando al Este, con el río Paraná; al Oeste, con el río Salado; al Norte, con la ciudad de Recreo y al Sur, con el río Santa Fe y el río Salado. La ciudad se ve altamente influenciada por su cercanía a estos ríos, los cuales suelen provocar inundaciones recurrentes en la región. En abril de 2003, la conjunción de la crecida del río Salado, la falta de obras y de planes de prevención, provocó más de 150 mil evacuados e innumerables daños y pérdidas al inundar la tercera parte de la ciudad; semejante inundación sólo tenía un precedente en 1905, pero en aquel momento fue el río Paraná. En marzo de 2007 la gran altura de ambos ríos influyó en la inundación de gran parte de la ciudad con miles de evacuados nuevamente.

El problema de las inundaciones y por ende de cómo se debe actuar en estas situaciones, nos lleva a tener que contar con mapas de riesgo; que son la representación gráfica de las características de un barrio o sector elegido, con información referente a las amenazas existentes, los diferentes grupos vulnerables y los recursos con los que cuenta la comunidad para enfrentar una emergencia por la manifestación de alguna de las amenazas identificadas.

El concepto de riesgo y el enfoque de los desastres han experimentado una evolución en las últimas décadas. Natezon (2006) al considerar las categorías de vulnerabilidad y riesgo, afirma que son fenómenos globales con características locales. Las evidencias presagian como escenarios futuros, el aumento extendido de la vulnerabilidad social con sus variables de peligrosidad y riesgo.

La cartografía de riesgo, instrumento capital para las políticas de prevención de desastres y reducción de sus consecuencias, se ha desarrollado con un predomino inicial de enfoques físicoestructurales (mapas de áreas geográficas, de localización de peligros y amenazas) en detrimento de la consideración del factor humano y la vulnerabilidad, dimensiones de más reciente incorporación (Ribera Masgrau, 2004).

La Conferencia Mundial sobre la Reducción de los Desastres (2005), al aprobar el Marco de Acción de Hyogo para 2005-2015, puso de relieve la necesidad de: "elaborar, actualizar periódicamente, definir y difundir mapas de riesgo...", como así también la de apoyar el mejoramiento de los métodos y capacidades científico tecnológicas de evaluación de riesgos mediante la investigación y la formación. Definimos a los mapas de riesgo como las representaciones gráficas de las características de un barrio o sector elegido, con información referente a las amenazas existentes, los diferentes grupos vulnerables y los recursos con los que cuenta la comunidad
2) Se trata del Curso de Posgrado de Actualización y Perfeccionamiento "Desastres, catástrofes y emergencias complejas. Salud Mental, vulnerabilidad y prevención (el riesgo de no prevenir el riesgo)", en 2006; Capacitación de grado para alumnos de la
Licenciatura en TO de la UNL, en la forma de un curso-taller y con amplia convocatoria.

3) Posgrado en la Especialidad en Terapia Ocupacional Comunitaria. Universidad Nacional de Quilmes. Seminario dictado: "Terapia Ocupaciona en situaciones de desastres". Octubre 2013. Buenos Aires. UNQ; Taller Capacitación Terapia Ocupacional en situaciones de desastres. La Plata. Mayo 2013. Actividad convocada por la Asociación de TO de la Provincia de Buenos Aires en respuesta a la inunda- ción que afectó a esa ciudad; Curso de Posgrado de Actualización y Extensión "Producción del conocimiento sobre catástrofe en la Universidad Nacional del Litoral”. 2008. UNL. 
para en $\neg$ frentar una emergencia por la manifestación de alguna de las amenazas identificadas.

Este recurso, constituye una herramienta para el diagnóstico en el proceso de gestión de riesgo comunitario. Es también un instrumento de planificación local para la acción, que surge del diagnóstico socializado por los diferentes actores locales. Permite determinar la naturaleza y las dimensiones de las pérdidas y el conocimiento de los peligros y la vulnerabilidad que configuran los riesgos.

La metodología de elaboración de mapas de riesgo está estrechamente ligada al enfoque teórico. La participación de la comunidad en esta tarea es particularmente recomendada y adoptada por organismos y gobiernos abocados al tema, comenzando por su elaboración a nivel local.

Los mapas pueden constituir instrumentos efectivos para mejorar la preparación de las poblaciones ante desastres. Por tratarse de un problema de salud pública, quienes trabajan en el área están llamados a unir esfuerzos y aportes multidisciplinares e intersectoriales para su elaboración.

De la forma como la comunidad se organice y prepare, dependerá en gran parte que pueda afrontar las emergencias y recuperarse. Asimismo, según las medidas que la comunidad implemente para reducir los riesgos, se logrará reducir los efectos; de allí la importancia de identificar los riesgos y organizar planes de emergencia. La generación del riesgo es un proceso social que tiene impacto en toda la comunidad, por lo tanto es fundamental asumir la corresponsabilidad en la gestión del riesgo, y comprender que todos, comunidad, instituciones, organizaciones, empresas y entidades, pueden contribuir a la solución.

A nivel local, tanto el gobierno de la ciudad como la Universidad, vienen desarrollando planes y proyectos de extensión, investigación y capacitación, pero su alcance no llega a toda la población comprometida en este tema, por lo que aparece la necesidad de trabajar en forma conjunta.

Se trata de una contribución al mejoramiento local que favorece la construcción de comunidades seguras desde la perspectiva del riesgo. En tanto se toma un problema que es propio del lugar y son los mismos actores involucrados en él quienes participan en la búsqueda de alternativas de solución.

En la ciudad de Santa Fe se ha establecido un Sistema Municipal de Gestión de Riesgos para dar un marco integral a las acciones tendientes a la reducción de riesgos. El sistema - creado por la
Ordenanza 11.512 en el año 2008 - es un espacio participativo destinado a trabajar en la prevención y mitigación de riesgos, en los preparativos para dar una mejor respuesta ante los eventos y en la recuperación posterior a emergencias o desastres.

\section{La intervención desde TO sobre riesgo}

El problema que acarrean las inundaciones como evento cíclico en una comunidad implica el cómo se debe actuar en estas situaciones, para lo que se torna necesario contar con mapas de riesgo. En el marco de este problema identificado y evidenciado por el Gobierno de la ciudad de Santa Fe a través de la Dirección de Gestión de Riesgo es que se planteó esta propuesta de trabajo. El propósito de ésta fue el de; promover desde la formación y la práctica académica en territorio, el desarrollo de aprendizajes de los estudiantes universitarios, con compromiso socio-comunitario y la contribución a la solución de problemas reales, favoreciendo de este modo el desarrollo de la responsabilidad ciudadana. Considerando el propósito de la práctica, se plantearon objetivos de aprendizajes, donde los alumnos que participan pueden: realizar diagnósticos y abordajes participativos en relación a la problemática y en escenarios reales; adquirir habilidades para detectar necesidades y problemas de la comunidad, relacionados con los riesgos y; desarrollar la capacidad de resolución de problemas con estrategias comunitarias. Con el énfasis puesto en que todos los participantes puedan: apreciar el valor y apropiarse constructivamente de los conocimientos relacionados con las medidas de prevención del riesgo.

La experiencia se desarrolló en el barrio Villa Oculta ${ }^{4}$, de Santa Fe, que está ubicado al oeste de la ciudad, lindero a un terraplén de defensa contra inundaciones que limita con el río Salado. El trabajo se realizó directamente con los pobladores del barrio elegido, con agentes municipales y con los estudiantes de las asignaturas participantes. Por lo que las instituciones del territorio fueron; La Dirección de Gestión de Riesgo del Gobierno de la ciudad de Santa Fe y la Organización no gubernamental comunitaria del barrio seleccionado "Movimiento los Sin Techo" 5

La metodología de trabajo estuvo centrada en elaborar una propuesta preliminar junto a la Dirección de Gestión de Riesgo del Gobierno de la ciudad de Santa Fe, los estudiantes como de la población, a los efectos de propiciar la reflexión y planificación participativa, en el momento previo al desarrollo de la práctica.
4) Villa Oculta es una estrecha lonja de terreno que, por su cercanía con el centro comercial de la ciudad, hace más de 45 años estas tierras fueron las elegidas por un grupo de personas dedicadas al cirujeo que echaron raíces $y$ levantaron sus viviendas.
5) La Organización No Gubernamental, El Movimiento Los Sin Techo, surge en el año 1987 fundada por el sacerdote, Padre Atilio Rosso, cuyo propósito, es la de proteger y avanzar en el derecho a la vivienda, el desarrollo del hábitat, el derecho a la vida y el derecho a la educación, de los sectores más vulnerables de nuestra ciudad. Este movimiento tiene un fuerte trabajo y una importante presencia en el barrio seleccionado para la experiencia desarrollada. Cabe aclarar que la organización fue invitada a formar parte del equipo, por la Dirección de Gestión de Riesgo, invitación que acordamos, considerando la significativa presencia en el barrio seleccionado. 
La metodología de taller y el diseño emergente se combinaron con el enfoque centrado en el grupo y en los contextos.

La integración docencia y extensión, se realizó a partir de las asignaturas y contenidos curriculares relacionados al tema en el que se trabajó. A saber:

Fundamentos, Métodos y Técnicas: Áreas de desempeño profesional. Área Comunidad: Análisis comunitario. Área emergente: ciclo de desastres. Prevención.

Estadística metodológica: Estadística descriptiva, tablas, gráficos, medidas de resumen, de posición y variabilidad, intervalos de confianza de parámetros como media y proporción.

Los criterios para la incorporación curricular fueron la participación en la presente propuesta en carácter de actividad optativa, el énfasis estuvo puesto en la decisión de compromiso de los alumnos y docentes con la comunidad.

El diseño de la propuesta tuvo la flexibilidad suficiente para que todos los alumnos participen de la gestión de ella, desde el inicio hasta la finalización y pudieran a la vez, elegir las actividades puntuales en las que desearon participar acorde a sus intereses e inquietudes.

La idea de integración como plantea Edith Litwin (2008) cuando dice:

"integrar, en la manifestaciones de los docentes, significa que los estudiantes doten de sentido al conocimiento adquirido.

Esto es que reconozcan el origen, su valor y su vinculación con otros temas o problemas. Pueden relacionarlo con el pasado histórico, con intereses sociales, con necesidades personales. En síntesis, integrar es, desde la perspectiva del conocimiento, relacionar" (Litwin, 2008:73)

Esta idea, nos remite a pensar: ¿cómo se aprende? ¿Cómo se hace para integrar lo que se sabe con lo que se debe aprender? Eso comprendiendo que conocer no es almacenar conceptos sino poderlos comprender, poder tener la posibilidad de construir conocimientos, entenderlos en sus contextos a partir de lo que se sabe con lo nuevo por aprender. Se trata de diseñar en función de que se recupere la implicación y la emoción. Un buen problema o tema puede permitir una cobertura curricular, además de darle significado al proceso de conocer y aprender.

¿Qué significa construir un mapa? Significa elaborar la representación gráfica de las amenazas y las vulnerabilidades de una comunidad, que puedan representar un riesgo para las personas. La elaboración de los mapas de riesgo es responsabilidad de la comunidad, la participación de esta es sumamente importante e imprescindible, porque sus integrantes son los que conocen el territorio, los recursos que existen, las potencialidades y las limitaciones locales. Para ello deben ser acompañados; y en este caso el acompañamiento fue por parte de la Universidad a través de esta práctica junto a la Dirección de Gestión de Riesgo de la Municipalidad.

Entre los actores claves que intervinieron en todo este proceso están: autoridades locales (personal profesional y técnico); los miembros de la ONG que prestan sus servicios en la comunidad; líderes/referentes locales, vecinos del barrio, docentes y estudiantes de la Universidad.

Considerando la problemática a abordar, y con la evidencia de que el Gobierno de la Ciudad de Santa Fe no ha incorporado el mapa de riesgo como una herramienta de gestión, creemos de suma importancia haber podido trabajar con ellos, la comunidad y los estudiantes de TO en la elaboración de las bases para la construcción de un mapa piloto.

\section{Conclusión}

A modo de conclusión, podemos decir que a partir de esta propuesta uno de los aspectos más significativos de la experiencia es la oportunidad que tuvieron los estudiantes de $1^{\circ}$ año de esta carrera de aprender un tema del programa, como es el "Análisis comunitario", el cual comprende un conjunto de contenidos tales como: perfil sociodemográfico de la población, recursos institucionales (humanos, materiales), políticas, servicios, historia, cultura, valores, problemas y necesidades. La identificación, relevamiento y análisis de estos datos en terreno y en contacto directo con la población generaron un proceso dialéctico entre la teoría y la práctica, con retroalimentación permanente. Esta relación también generó por momentos algunas tensiones que produjeron revisiones y reflexiones propias de la construcción colectiva y contextuada de los conocimientos, y a partir de ello se pudo comprender y aprender de otra forma, relacionar conocimientos y contenidos en un escenario real, con situaciones concretas y reales, teniendo la posibilidad de pensar en estrategias comunitarias con los propios vecinos del barrio. En palabras de E. Litwin: "la significatividad social se construye al entender los conocimientos en una trama de relaciones o vínculos, tal como se presentan en el mundo científico y en el acontecer cotidiano" (2008:75).

Esto -creemos- quedó demostrado también en la participación y los resultados de las evaluaciones realizadas por los estudiantes en otra asignatura ${ }^{6}$ de cursado paralelo a esta experiencia, donde se reflejó en la reflexión hecha por éstos el aprendizaje realizado en la misma.
6) Asignatura: Psicología del desarrollo ubicada en el $2^{\circ}$ cuatrimestre del $1^{\circ}$ año de la carrera de Licenciatura en Terapia Ocupacional. 
Otro de los aspectos tiene que ver con lo que han expresado los propios alumnos en cuanto a su participación, lo que les permitió, por ejemplo, cambiar y/o repensar en determinados prejuicios y miradas que tenían con relación al barrio y a la realidad de estos vecinos. La adaptación de los alumnos al territorio fue de manera progresiva para algunos e inmediata para otros. Esto se observó en el modo y las formas en que se desempeñaron y desenvolvieron en los dos primeros ingresos al barrio y lo que fueron los otros restantes, donde no tenían "miedo" o "vergüenza" de preguntar y/o interactuar con los vecinos.

También la oportunidad de vivenciar lo que puede ser uno de los campos de trabajo como licenciados en TO: el espacio comunitario. Y descubrir lo que significa el ejercicio profesional como compromiso y servicio a la comunidad. Así, los valores que estuvieron presentes fueron el compromiso con el trabajo, la responsabilidad, el respeto por el otro, la solidaridad, la capacidad de escucha.

Como toda práctica de extensión, estuvo atravesada por la realidad social del momento actual en el que se desarrolló, lo que produjo algunas dificultades. La más importante tuvo que ver con el intento de desalojo que sufrieron algunos vecinos en la fecha que debíamos ingresar al barrio. Este hecho provocó que uno de los grupos que fueron al barrio no pudiera ingresar al lugar del conflicto por sugerencia de la referente con la que se trabajó y acompañó en el mismo, cuestión que fue salvada en otras visitas sin problema alguno, por lo que se pudo seguir con los encuentros, entrevistas y recorrido.

Otra fue al momento de convocar para la jornada de intercambio, donde la participación de los agentes municipales fue escasa ya que debido al conflicto pidieron no intervenir para —según sus expresiones- no entorpecer el trabajo o para que no hubiera interpretaciones confusas de los vecinos, por lo que la convocatoria fue a través de la referente del barrio.

Cabe resaltar que los datos e información surgidos en esta prueba piloto con los vecinos del barrio y los agentes municipales quedaron a disposición y conocimiento de ambos. Asimismo, se realizó una Jornada de reflexión donde se intercambiaron los aprendizajes, las vivencias, los procesos y resultados obtenidos de este proceso en la que participaron todos los actores intervinientes de la propuesta. Todo el material se transformó en insumo para continuar la construcción del mapa en dicho barrio. De este modo, tal como lo plantea el Tecnológico de Monterrey: "estudiantes, docentes y miembros de la comunidad, trabajan y aprenden juntos para solucionar necesidades específicas de la comunidad, utilizando y transfiriendo el conocimiento para el bien común" (2006:3).

\section{Referencias bibliográficas}

Blesedell, E.; Scohn, E. y Boyt, B. (2005). Willard \& Spackman. Terapia Ocupacional. Buenos Aires: Panamericana.

Camilloni, A. (2010). Material Curso de Posgrado. "Las prácticas académicas en espacios socio culturales, productivos y/o comunitarios. Pensando la educación experiencial". Co-organizado por Facultad de Humanidades y Ciencias, Secretarías Académica y de Extensión UNL (Área de Incorporación Curricular de la Extensión). Estrategia Internacional para la Reducción de Desastres (EIRD) (2005). "Marco de Acción de Hyogo para 2005-2015: aumento de la resiliencia de las naciones y las comunidades ante los desastres". Conferencia Mundial sobre Reducción de Desastres. Naciones Unidas. (Ginebra, Suiza). 7. Disponible en: www.unisdr.org De Alba, A. (1995). Curriculum: crisis, mitos y perspectivas. Buenos Aires: Miño y Dávila.

Litwin, E. (2008). El oficio de enseñar. Condiciones y contextos. $1^{\circ}$ edición. Buenos Aires: Paidós.

Natezon, C. (2006). Vulnerables frente al riesgo. ConCiencia, 12(16), 10-11. Santa Fe: Ediciones Secretaría de Ciencia y Técnica de la Universidad Nacional del Litoral. Ribera Masgrau, L. (2004). Los mapas de riesgo de inundaciones: representación de la vulnerabilidad y aportación de las innovaciones tecnológicas. Doc. Anàl. Geogr. 43, 153-171.

Tecnológico de Monterrey. (2006). El modelo educativo del Tecnológico de Monterrey. Monterrey: Tecnológico de Monterrey.

UNL (2010). Plan de Desarrollo Institucional UNL (2010-2019). 